\section{EUS and EMR/ESD: Is EUS in patients with Barrett's esophagus with high-grade dysplasia or intramucosal adenocarcinoma necessary prior to endoscopic mucosal resection?}

\section{Waxman}

Department of Psychiatry, School of Medicine, University of Occupational and Environmental Health, Fukuoka, Japan

The management of patients with HGD in BE remains controversial, largely because the natural history of this condition is so poorly defined. For example, Reid et al. reported a 59\% 5-yr cumulative cancer incidence among 76 patients with HGD in BE [1],whereas Schnell et al. found that only 12 (16\%) of their 75 patients with HGD developed adenocarcinoma during a mean follow-up period of $7.3 \mathrm{yr}$ [2]. Four management options have been proposed for patients with HGD in BE: (1) esophagectomy, (2) endoscopic ablative therapies, (3) EMR, and (4) intensive endoscopic surveillance, in which invasive therapy is witheld until biopsy specimens show evidence of invasion [3]. Clearly, there are risks associated with all of these approaches, and all would benefit from accurate staging of the neoplasia.

\section{Estimation of the depth of tumor penetration}

The less than perfect reliability of endoscopic staging with the adjunct of chromo endoscopy and magnification endoscopy for the estimation of cancer depth before EMR can be improved by the use of endoscopic ultrasonography (EUS), in particular highfrequency US probe sonography (HFUPS). HFUPS may distinguish nine layers within the wall of GI organs in contrast to a five-layered structure seen with conventional EUS, thus providing better images that are useful in the evaluation of transmural penetration and in differentiating cancers limited to the mucosa from those with submucosal penetration. The main limitation of HFUPS is its tendency to overstage early lesions. The diagnostic accuracy of HFUPS in assessing depth of tumor invasion in the esophagus, stomach and colon ranges from $67 \%$ to $94 \%$ among the published studies [4-9]. This high variability in HFUPS accuracy may reflect the use of probes with different penetration $(15 \mathrm{MHz}$ versus $20 \mathrm{MHz})$ and differences in the patient populations studied. Accuracy of HFUPS, in fact, is significantly better for elevated type lesions than for depressed ones [7].

When different techniques are used in combination to select lesions suitable for EMR, the overall accuracy is high [10]. Because of the limitations of these staging techniques, however, it has

Correspondence: I. Waxman, M.D. · University of Chicago Hospitals · 5758 S. Maryland Avenue, MC 9028 - Chicago, IL 60637, USA · Phone/Fax: 001-201997-6610 · E-mail: iwaxman@medicine.bsd.uchicago.edu

Bibliography: Endoscopy 2006; 38 (S1): S2-S4 @ C Georg Thieme Verlag KG Stuttgart · New York · ISSN 0013-726X · DOI 10.1055/s-2006-946641 been suggested that when a lesion meets the generally accepted criteria of size, and appearance is encountered, EMR can be performed without prior HFUPS, as long as the lesion can safely be removed in its entirety [11-12]. Submucosal injection used to facilitate EMR can also help to decide whether or not to continue with the procedure [13]. The observation of a bleb formation with elevation of the overlying mucosa indicates the absence of deep submucosal involvement and the feasibility of EMR [30] On the other hand, the dense fibrosis associated with deep submucosal invasion prevents fluid infiltration through the submucosal connective tissue, decreasing bleb formation and elevation of the lesion [14]. This so-called "non-lifting sign has been found to have $100 \%$ sensitivity, $99 \%$ specificity, and $83 \%$ positive predictive value for invasive carcinoma in patients with early cancer of the colon [15]. Ultimately, depth of tumor invasion can be precisely established by histological analysis of EMR specimens, which in fact, is part of the diagnostic algorithm for the evaluation of early gastric cancer [16], and as recently suggested for high-grade dysplasia (HGD) and early adenocarcinoma arising in Barrett's esophagus (BE) [17].

\section{EUS in BE with HGD, ICA and early adenocarcinoma}

Falk et al. studied the utility of EUS in nine patients with BE and HGD or ICA using the conventional echo-endoscope (7.5 and 12 $\mathrm{MHz}$ ) [18]. In the six patients with HGD, EUS correctly predicted the absence of tumor in four, but incorrectly predicted the presence of tumor in two. EUS correctly predicted that there was tumor in one of the three patients with

ICA, but EUS overstaged that lesion. Indeed, EUS correctly staged only one of the three patients with ICA. The tendency of EUS to overstage was attributed primarily to inflammation in the wall of the esophagus. The authors also proposed that overstaging could have been caused by artifacts, such as overlapping folds pulled up by the balloon, or tangential imaging of the esophageal wall that makes it appear thicker on one side. Such artifacts appeared to occur most frequently around the gastroesophageal junction. Based on the above data, the authors concluded that conventional EUS was unable to distinguish ICA from HGD reliably in BE, and unable to stage early neoplasia accurately. The authors suggested that better delineation of the esophageal wall with HFPUS might provide more accurate information. Srivastava and colleagues reported their prospective experience with conventional EUS (12 MHz) in the evaluation of 17 patients with BE (6 with dysplasia) and 13 controls [19]. Patients with BE in general had greater esophageal wall thickness than controls. In two of the six patients with HGD, focal submucosal thickening was identified, and invasive T1b adenocarcinomas were identified in esophagectomy specimens.

Based on these results, the authors suggested that EUS may help in selecting patient's with HGD who may benefit from surgical resection. However, the conclusions that can be drawn from that study are limited because the authors did not specify the grade of dysplasia in four of the six patients in the dysplastic group, and surgical results are not provided in another four cases. Hence, the accuracy of EUS in this small number of patients cannot be ascertained. 
Scotiniotis et al. studied the accuracy of conventional EUS (7.5$12 \mathrm{MHz}$ ) in the evaluation of BE with HGD or ICA in 22 patients [20]. The sensitivity and specificity of EUS for detecting submucosal tumor invasion were $100 \%$ and $94 \%$, respectively. However, $55 \%$ of their patients had endoscopically apparent mucosal lesions or strictures. Similar to our results, EUS resulted in one false-positive diagnosis. Like Falk et al. [18], these authors also speculated that HFPUS might provide more accurate information. HFPUS with $20-\mathrm{MHz}$ probes provides finer resolution of the esophageal wall than conventional EUS. In contrast to the five-layered gastrointestinal wall structure seen with conventional EUS, HFPUS reveals 7-9 layers. There is better definition of the mucosa with clearer visualization of the muscularis mucosae, features that conceivably might result in better staging of neoplasia in BE [21,22]. Adrain et al. used HFPUS to evaluate 17 patients with $\mathrm{BE}$ ( 3 with dysplasia) and 12 control subjects [22]. $\mathrm{BE}$ was diagnosed when the second hypoechoic layer was thicker than the first hyperechoic layer of the mucosa and, using these criteria, HFPUS was 100\% accurate in distinguishing patients with $\mathrm{BE}$ from normal. However, the investigators identified no specific sonographic finding for dysplasia. They concluded that HFPUS is a sensitive tool for identifying Barrett's epithelium but not for detecting dysplasia. In a study by Waxman et, al. HFPUS was not highly accurate for detecting invasive esophageal cancer in patients with BE and HGD or ICA (23). As in the study by Adrain et al. [22], the authors found that HFPUS showed thickening of the mucosal sonographic layers of the esophagus in all nine of our patients. Although there was some correlation between the HFPUS and surgical pathology findings in six patients (67\%), HFPUS mistakenly diagnosed cancer in one patient (11\%) and missed cancers in two (22\%). In one of the two patients with a missed cancer by HFPUS, the error might have been due to the location (gastroesophageal junction) and small size $(2 \mathrm{~mm})$ of the cancer [23]. This suggests that, even at $20 \mathrm{MHz}$, HFPUS does not have the resolution needed to identify superficial cancers reliably. Similar experience was reported in abstract form by Parent et al. in 13 patients with HGD [24]. Thickening of layer two was noted in all patients studied, but HFPUS could not detect adenocarcinomas in the absence of endoscopically visible lesions.

Furthermore, in patients with biopsy-proven ICA, a normal sonographic examination could not exclude the presence of submucosal carcinoma [24]. Finally, in one of the largest recent studies dealing with the question of HFUPS in early cancer in BE by May et.al.[25], only two of 14 patients with submucosal tumor invasion diagnosed after EMR had been correctly identified beforehand. Furthermore, endoscopic assessment of tumor penetration was as good as HFUPS.

Two recent published studies dealing with malignant adenopathy in the presence of ICA and early adenocarcinoma support revisiting the role of EUS in the evaluation on patients who are being considered for endoscopic therapy $[26,27]$. Although the studies have many limitations, further research into the role EUS may play in the management algorithm of patients being considered for endoscopic therapy is warranted [28].

In summary, based on available data, HFUPS appears to have limited value in accurately predicting depth of penetration of early adenocarcinoma in $\mathrm{BE}$ and hence cannot be the sole factor in deciding a therapeutic conduct. A detailed endoscopic assessment provides invaluable information and should be undertaken prior to EMR in patients with BE with ICA or early adenocarcinoma. The role of EUS in excluding malignant adenopathy remains to be determined.

\section{References}

${ }^{1}$ Reid BJ, Levine DS, Longton G et al. Predictors of progression to cancer in Barrett's esophagus: Baseline histology and flow cytometry identify low- and high-risk patient subsets. Am J Gastroenterol 2000; 95: $1669-1676$

2 Schnell TG, Sontag SJ, Chejfec G et al. Long-term nonsurgical management of Barrett's esophagus with high-grade dysplasia. Gastroenterology 2001; 120: 1607-1619

${ }^{3}$ Spechler SJ. Dysplasia in Barrett's esophagus: Limitations of current management strategies. Am J Gastroenterol 2005; 100: 927 - 935

${ }^{4}$ Saitoh Y, Obara T, Einami K, Nomura M, Taruishi M, Ayabe T et al. Efficacy of high-frequency ultrasound probes for the preoperative staging of invasion depth in flat and depressed colorectal tumors. Gastrointest Endosc 1996; 44 (1): $34-39$

${ }^{5}$ Murata Y, Suzuki S, Ohta M, Mitsunaga A, Hayashi K, Yoshida K et al. Small ultrasonic probes for determination of the depth of superficial esophageal cancer. Gastrointest Endosc 1996; 44 (1): 23-28

${ }^{6}$ Akahoshi K, Chijiiwa Y, Hamada S, Harada N, Nawata H. Endoscopic ultrasonography: a promising method for assessing the prospects of endoscopic mucosal resection in early gastric cancer. Endoscopy 1997; 29 (7): 614-619

${ }^{7}$ Akahoshi K, Chijiwa Y, Hamada S, Sasaki I, Nawata H, Kabemura T et al. Pretreatment staging of endoscopically early gastric cancer with a 15 $\mathrm{MHz}$ ultrasound catheter probe. Gastrointest Endosc 1998; 48 (5): 470-476

${ }^{8}$ Murata Y, Suzuki S, Mitsunaga A, Iizuka Y, Uchiyama M, Uchida K et al. Endoscopic ultrasonography in diagnosis and mucosal resection for early esophageal cancer. Endoscopy 1998; 30 (Suppl 1): A44-46

${ }^{9}$ Kida M, Tanabe S, Watanabe M, Kokutou M, Kondou I, Yamada Y et al. Staging of gastric cancer with endoscopic ultrasonography and endoscopic mucosal resection. Endoscopy 1998; 30 (Suppl 1): A64-68

${ }^{10}$ Yanai H, Matsumoto Y, Harada T, Nishiaki M, Tokiyama H, Shigemitsu T et al. Endoscopic ultrasonography and endoscopy for staging depth of invasion in early gastric cancer: a pilot study. Gastrointest Endosc 1997; 46 (3): $212-216$

${ }^{11}$ Rembacken BJ, Gotoda T, Fujii T, Axon AT. Endoscopic mucosal resection. Endoscopy 2001; 33 (8): 709-718

12 Soetikno RM, Gotoda T, Nakanishi Y, Soehendra N. Endoscopic mucosal resection. Gastrointest Endosc 2003; 57: 567 (4) - 79

${ }^{13}$ Kato H, Haga S, Endo S, Hashimoto M, Katsube T, Oi I et al. Lifting of lesions during endoscopic mucosal resection (EMR) of early colorectal cancer: implications for the assessment of resectability. Endoscopy 2001; 33 (7): $568-573$

${ }^{14}$ Ishiguro A, Uno Y, Ishiguro Y, Munakata A, Morita T. Correlation of lifting versus non-lifting and microscopic depth of invasion in early colorectal cancer. Gastrointest Endosc 1999; 50 (3): 329-333

${ }^{15}$ Uno Y, Munakata A. The non-lifting sign of invasive colon cancer. Gastrointest Endosc 1994; 40 (4): 485-489

16 Tada M. Endoscopic mucosal resection of the stomach: initial description. Gastrointest Endosc Clin N Am 2001; 11 (3): 499-510

${ }^{17}$ Lightdale CJ, Larghi A, Rotterdam H, Okpara N. Endoscopic ultrasonography (EUS) and endoscopic mucosal resection (EMR) for staging and treatment of high-grade dysplasia (HGD) and early adenocarcinoma (EAC) in Barrett"s esophagus (BE). Gastrointest Endosc (abstract AB90(, 2004; 59(5)

${ }^{18}$ Falk GW, Catalano MF, Sivak MVJr et al. Endosonography in the evaluation of patients with Barrett's esophagus and high-grade dysplasia. Gastrointest Endosc 1994; 40: 207-212

${ }^{19}$ Srivastava AK, Vanagunas A, Kamel P et al. Endoscopio ultrasound in the evaluation of Barrett's esophagus: A preliminary report. Am J Gastroenterol 1994; 89: 2192-2195

${ }^{20}$ Scotiniotis IA, Kochman ML, Lewis JD et al. Accuracy of EUS in the evaluation of Barrett's esophagus and high-grade dysplasia or intramucosal carcinoma. Gastrointest Endosc 2001; 54: 689-696 
${ }^{21}$ Kimmey MB, Martin RW, Silverstein FE. Endoscopic ultrasound probes. Gastrointest Endosc 1990; 36: S40-46

22 Adrain AL, Ter HC, Cassidy MJ et al. High-resolution endoluminal sonography is a sensitive modality for the identification of Barrett's metaplasia. Gastrointest Endosc 1997; 46: 147 -151

${ }^{23}$ Waxman I, Raju GS, Critchlow J et al. High-Frequency Probe Ultrasonography Has Limited Accuracy for Detecting Invasive Adenocarcinoma in Patients with Barrett's Esophagus and High-Grade Dysplasia or Intramucosal Carcinoma: A Case Series. Am J of Gastroenterol, 2006: (in press)

${ }^{24}$ Parent J, Levine DS, Haggitt RC et al. Role of endoscopic ultrasound in patients with Barrett's esophagus and high grade dysplasia. Gastrointest Endosc 1997; 45: A76

${ }^{25}$ May A, Gunter E, Roth F et al. Accuracy of staging in early oesophageal cancer using high resolution endoscopy and high resolution endosonography: A comparative, prospective, and blinded trial. Gut 2004; 53: $634-640$

${ }^{26}$ Shami V, Villaverde A, Stearns L et al. Impact of EUS and EUS-FNA in patients with Barrett's Esophagus and High-grade dysplasia or intramucosal adenocarcinoma referred for endoscopic therapy.. Endoscopy 2006; 38: 157 - 161

${ }^{27}$ Bollschweiler E, Baldus SE, Schroder W et al. High rate of lymph-node metastasis in submucosal esophageal squamous-cell carcinoma and adenocarcinoma. Endoscopy 2006; 38: 149-156

${ }^{28}$ Vieth M, Rosch T. Endoscopic mucosal resection and the risk of lymphnode metastases: Indications revisited. Endoscopy 2006; 38: 175 - 179 